\title{
Shock induced detonations in composite heterogeneous energetic materials
}

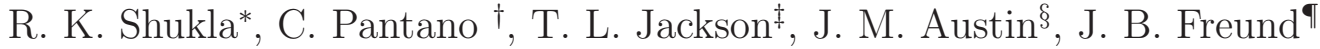 \\ University of Illinois at Urbana-Champaign, Urbana, IL, 61801
}

\begin{abstract}
Solid energetic materials are used in a wide variety of applications, including solid rocket motors, munitions, explosives for construction and demolition, automotive airbags, and pyrotechnic fasteners and actuators for space applications. An understanding of their potential for initiation and explosion is vital for their safe storage, handling, and transportation. Because of the rich phenomenology associate with microstructural geometric features, such pack as morphology, the presence of voids, and the type of binder, one-dimensional empirical models will be limited in predicting the shock sensitivity of energetic materials for a wide variety of insults. Therefore, the goal of our research is to develop a multidimensional shock-sensitivity model that accounts for microstructural geometric features. Here, we report a novel shock capturing multi-phase flow solver, which combines the features of a level-set method and a time dependent mesh redistribution technique. Numerical simulations of multi-material inert shock problems and detonation initiation through localized thermal energy deposition are used to demonstrate the method.
\end{abstract}

Shock capturing methods are widely used in simulation of compressible multi-fluid reacting flows. A major drawback of these methods is that field representations of multi-material interfaces suffer from numerical smearing even though shocks are captured within a few grid cells. ${ }^{1,2}$ In our approach, special attention is paid to minimizing numerical diffusion of multi-material interfaces. In order to allow for dynamic creation of interfaces, particularly when reactants convert into products, we have chosen to employ the diffuse interface framework. ${ }^{3}$ However, we have utilized an interface compression technique in order to minimize the diffusion of multi-material interfaces. Like shocks, material interfaces are captured across a few grid cells. ${ }^{4}$ Additionally we utilize an adaptive mesh redistribution technique, developed originally for hyperbolic conservation laws, ${ }^{5,6}$ in order to focus computational resources close to sharp gradients. This is especially attractive for reacting flows since adaptive mesh redistribution enables resolution of thin combustion zones which remain under-resolved on a uniform grid. Further details of the numerical methods and simulation procedures are reported elsewhere. ${ }^{4}$

To demonstrate the basic numerical methodology we show that our code is able to simulate both a onedimensional and a two-dimensional shock to detonation transition using the initiation model of Sileem et al. ${ }^{7}$ and Regele et al. ${ }^{8,9}$ We assume that the reacting system is adequately modeled by the Euler equations with one-step Arrhenius kinetics, as has been done in the past to study deflagration to detonation transition. ${ }^{7-9}$ Despite its superficial simplicity, this model system involves a wide range of spatial and temporal scales, which challenge numerical simulation. The Euler equations are augmented with source terms that model both external thermal heat addition and combustion heat release. These are supplemented with an additional equation for mass fraction of the unburnt species. A second-order Strang splitting is employed to circumvent numerical stiffness caused by the disparate transport and reactive time scales. ${ }^{1}$ A high-resolution Godunov method is used along with the structure of the Riemann solution to determine where combustion should occur at each time step of the computation.

Our reacting solver is demonstrated on the one-dimensional benchmark detonation initiation problem of Regele et al. ${ }^{8}$ in which a time dependent heat release initiates the reaction. Figure 1 shows pressure as a

\footnotetext{
${ }^{*}$ Postdoc, Computational Science and Engineering, University of Illinois

$\dagger$ Assistant Professor, Mechanical Science and Engineering, Member AIAA

${ }^{\ddagger}$ Senior Scientist, Computational Science and Engineering, Associate Fellow AIAA

$\S$ Assistant Professor, Aerospace Engineering, Member AIAA

『Associate Professor, Departments of Mechanical Science and Engineering and Aerospace Engineering, jbfreund@illinois.edu, Senior Member AIAA
} 

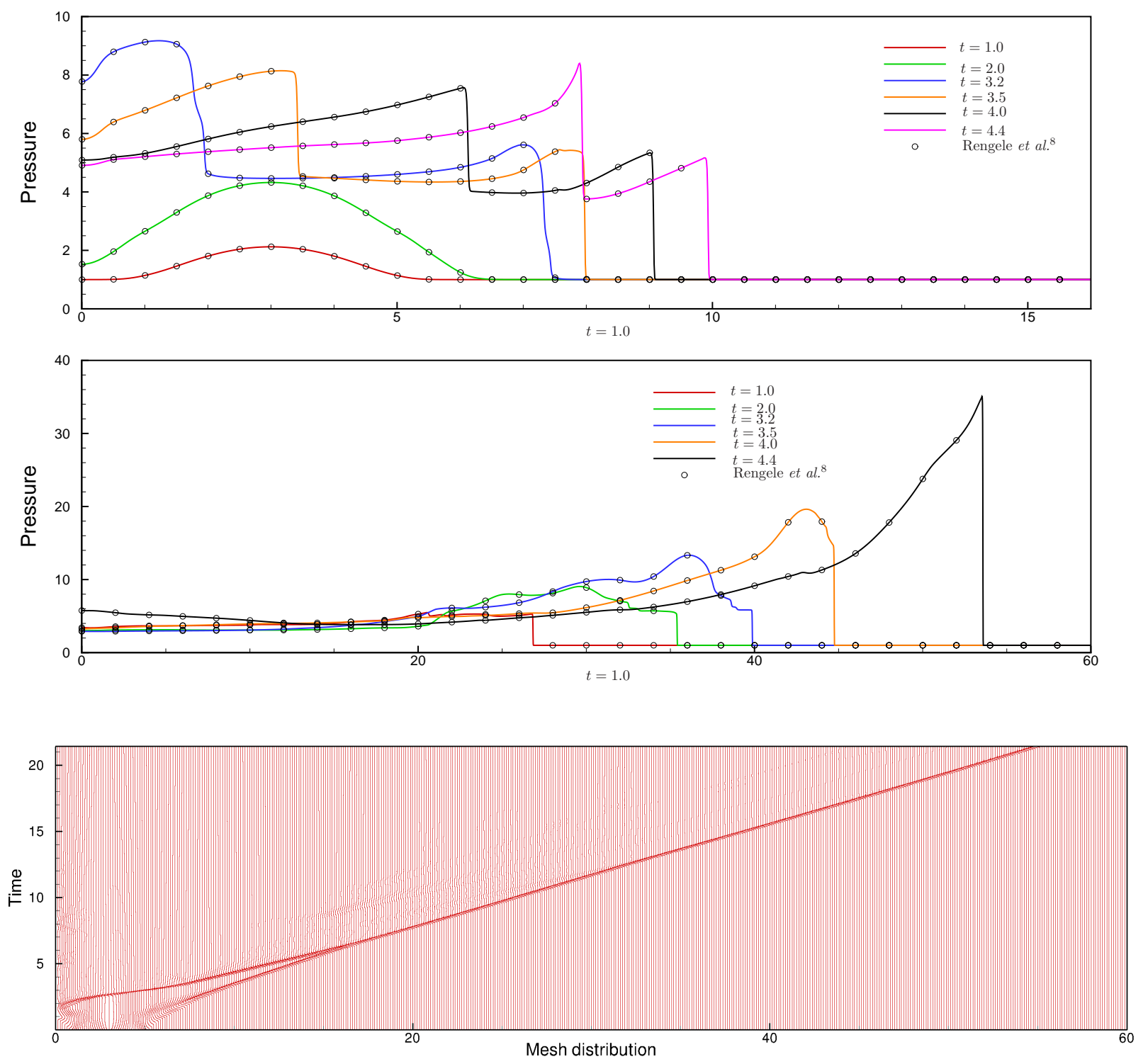

Figure 1. Pressure (top and center) and mesh distribution (bottom) as a function of time for one-dimensional detonation initiation problem. Results of Regele $e t a .^{8}$ are denoted by (०).

function of time plotted against results of Regele et al. ${ }^{8}$ The agreement is excellent. We have extended the one-dimensional computational methodology to two dimensions using dimensional splitting and adaptive mesh redistribution. Results for the two-dimensional detonation initiation problem of Regele et al. ${ }^{9}$ are shown in figure 2 along with the time dependent adaptive grid.

We have combined the reaction capability with the multi-material methods discussed in order to simulate the entire processes of detonation initiation in a propellant. This is an important step towards our ultimate goal of achieving truly predictive capability of shock-to-detonation transition in complex energetic materials. In order to probe the influence of heterogeneities on the shock sensitivity of energetic materials we consider the computational setup shown in figure 3, which consists of a shock traversing a model solid propellant pack comprised of HMX and AP in an estane binder. In this simulation the top and bottom boundaries are periodic. All the components are treated as separate phases and are modeled using a stiffened equation of state, with constants chosen in order to match their respective shock Hugoniots. An initial $M=2.28$ shock wave in estane, which corresponds to a shock pressure ratio of $5 \times 10^{4}$, is seen at the left side of the domain in figure 3. The products are assumed to follow an ideal gas equation of state with $\gamma=1.2$. Real gas effects and finite thermal conductivity of the heterogeneous medium are ignored in these calculations. 

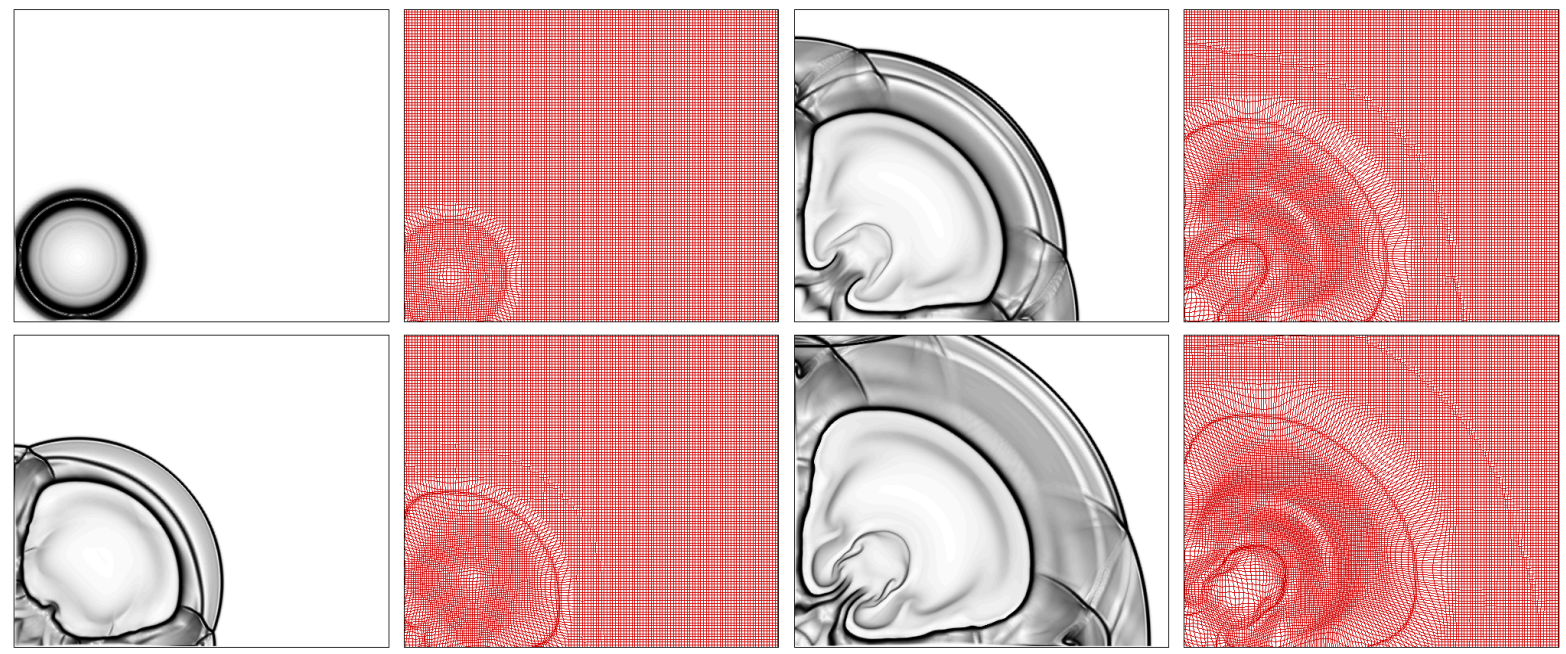

Figure 2. Density gradient contours and adaptive grid for two-dimensional thermal detonation initiation problem. Computations are performed on a $1500 \times 500$ adaptive grid.

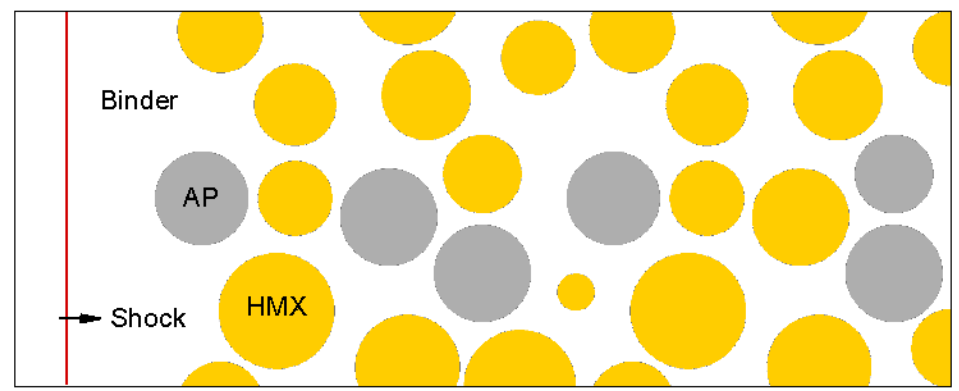

Figure 3. Schematic of a shock propagating through a heterogeneous energetic material.

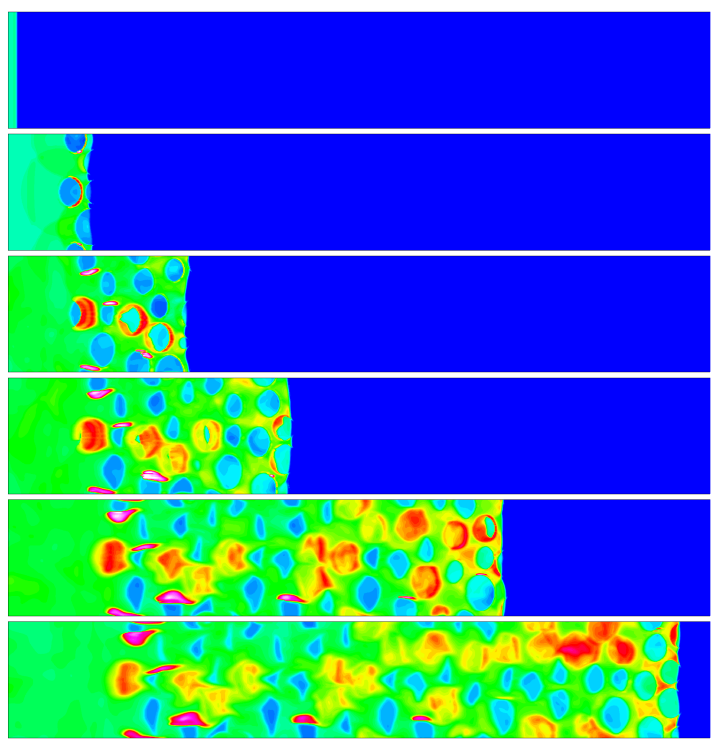

(a) Isotherms with blue to red contours depicting temperature variation from $300 \mathrm{~K}$ to $1800 \mathrm{~K}$
Figure 4(a) depicts temperature as a function of time. We observe that shock focusing due to heterogeneities leads to high local temperatures. While these high temperatures trigger combustion of AP they are still relatively low so that HMX is not immediately ignited. This observation is confirmed from the phase-field visualization in figure 4(b), where combustion of AP (yellow) leads to formation of gaseous products (blue) whereas the HMX crystals (red) are not ignited.

Figure 4. Isotherms and phase field contours for shock propagation through HMX AP model propellant computed on a $1600 \times 200$ computational grid. 


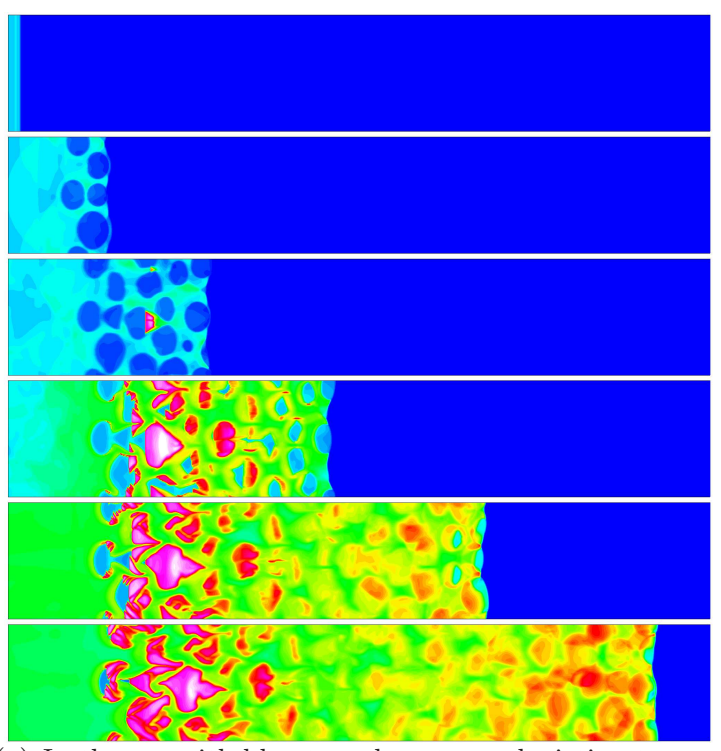

(a) Isotherms with blue to red contours depicting temperature variation from $300 \mathrm{~K}$ to $4800 \mathrm{~K}$

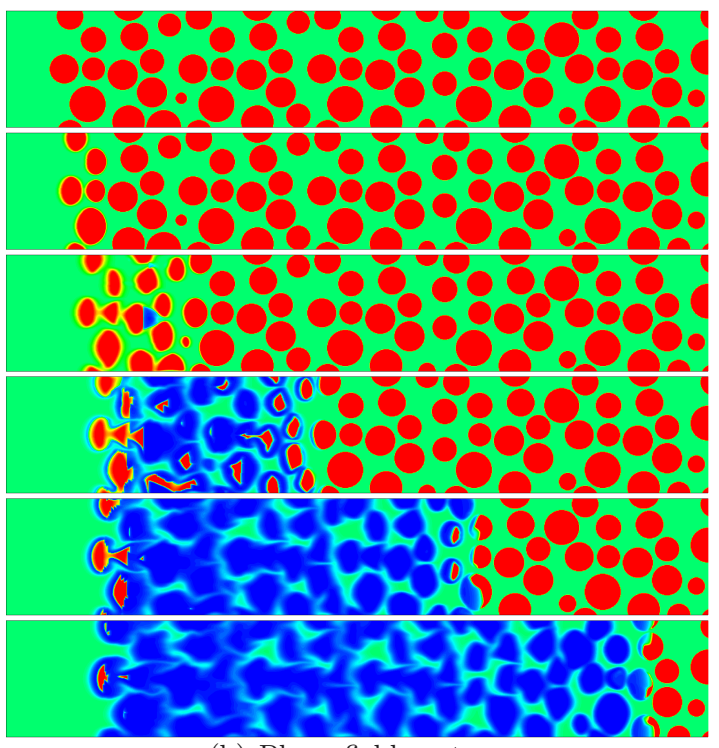

(b) Phase field contours

Figure 5. Isotherms for shock propagation through HMX model propellant computed on a $1600 \times 200$ computational grid.

A second computation is done with the same configuration except that now all the AP is replaced with HMX. The the temperature and phase fields are visualized in 5(a). Here localized shock focusing leads to ignition and formation of a detonation wave which eventually overtakes the lead shock. As shown in figure 5(b), this detonation wave consumes HMX and converts it into gaseous product as it traverses through the propellant.

In conclusion, we have introduced a multi-component reacting compressible flow solver, which utilizes interface compression and a adaptive moving mesh technique in order to achieve high accuracy when modeling the material extent on by field variables. The application of this technique has been demonstrated on benchmark one-dimensional and two-dimensional detonation initiation problems. Finally the importance of distribution of inhomogeneities in shock-to-detonation transition in heterogeneous propellants has been highlighted through simulations which showed formation of a detonation wave in a propellant composed of HMX and estane but not in the one with AP as an additive.

This work was supported by the US Department of Energy via the Center for Simulation of Advanced Rockets at the University of Illinois at Urbana-Champaign and by the United States Air Force Office of Scientific Research.

\section{References}

${ }^{1}$ Leveque, R. J. Finite volume methods for hyperbolic problems. Cambridge University Press, Cambridge (2002).

${ }^{2}$ Toro, E. F. Riemann solvers and numerical methods for fluid dynamics, second ed., Springer (1999).

${ }^{3}$ Abgrall, R., Karni, S. Computations of compressible multifluids. J. Comput. Phys., 169, 594-623 (2001).

${ }^{4}$ Shukla, R. K., Pantano, C. A., Freund, J. B. An adaptive moving mesh method for compressible multiphase flows. Under Preparation.

${ }^{5}$ Tang, H., Tang, T. Adaptive mesh methods for one- and two-dimensional hyperbolic conservation laws. SIAM J. Numer. Anal., 41, 487-515 (2003).

${ }^{6}$ Azarenok, B. N., Tang, T. Second-order Godunov-type scheme for reactive flow calculations on moving meshes. J. Comput. Phys., 206, 48-80 (2005).

${ }^{7}$ Sileem, A. A., Kassoy, D. R., Hayashi, A. K. Thermally initiatied detonation through deflagaration to detonation transition. Proc. R. Soc. Lond. A, 435, 459-482 (1991).

${ }^{8}$ Regele, J. D., Kassoy, D. R., Vasilyev, O. V. Detonation initiation on the microsecond timescale: one and two dimensional results obtained from adaptive wavelet-collocation numerical methods. AIAA 2007-986 (2007).

${ }^{9}$ Regele, J. D., Kassoy, D. R., Vasilyev, O. V. Numerical modeling of acoustic timescale detonation initiation. AIAA 2008-1037 (2008). 